\title{
Structure et sens en musique : dialogue avec Greimas
}

\section{INTRODUCTION}

Au sein des sciences humaines, la musicologie correspond à l'étude scientifique de la musique. L'exploration de l'évolution des styles musicaux dans la musique occidentale se fait, le plus souvent, à l'aide de l'examen des genres musicaux (Danuser 1998). Le genre musical offre un cadre prédéterminé pour la musique du point de vue de son effectif et de sa forme, mais aussi de sa fonction sociale et de son contenu. Le philosophe et l'esthéticien hongrois de la musique, J. Ujfalussy décrit ce rapport dialectique de la manière suivante :

Le genre communique vers le sujet d'une part, et vers la structure, d'autre part. La dialectique entre les deux, créée dans et par la musique, est une particularité qui mérite un examen approfondi. [...] Le genre tourne une de ses faces vers le sujet (le thème, le contenu). Ce dernier dépend de manière directe des changements de circonstances de la vie collective, il les suit de façon immédiate. Ces changements poussent et obligent le genre à se métamorphoser perpétuellement. L'autre face du genre est de nature structurelle. La structure musicale garde et conserve, avec une force particulière, ses traits fondamentaux : elle attacherait, immobiliserait le genre en empêchant ses transformations. En dernier lieu, c'est toujours l'impulsion venant du côté du sujet qui l'emporte. Ce dernier (le thème, le sujet) transforme le contenu et, avec lui, les traits structurels et stylistiques du genre. (Ujfalussy, 1968 : 151)

Par la suite, nous examinerons ces deux versants d'une pièce ou d'un genre musical : la structure et le sens. 


\section{LE RÔLE DE LA STRUCTURE (ET DE LA FORME) EN MUSIQUE}

Il n'existe pas de musique sans structuration, les règles des formes sont définies depuis que le concept $\mathrm{d}^{\prime}$ « œuvre musicale » existe ${ }^{1}$.

Étant donné que la question de la forme ou de la structure est inhérente à la réflexion musicale, la définition de la forme donnée par A. J. Greimas et J. Courtés éclaire considérablement le problème de la musique également, tout en rimant avec la définition de J. Ujfalussy :

Les emplois divers et variés du mot forme reflètent pratiquement toute l'histoire de la pensée occidentale. [...] En effet, la notion de "forme » a hérité de la tradition aristotélicienne sa place éminente dans la théorie de la connaissance : opposée à la matière qu' elle 'informe', tout en 'formant' l'objet connaissable, la forme est ce qui garantit sa permanence et son identité. Dans cette acception fondamentale, la forme, on le voit, est proche de notre conception de structure. (Greimas \& Courtés, 1979 : 155)

Dès 1971, A. J. Greimas précise que " toute la science exacte est naturellement structuraliste, parce qu'elle ne peut pas être différente » (Greimas, [1971] 2017 : 40). La musique n'est pas nécessairement une science exacte, sauf si on l'examine du point de vue de sa construction physique (acoustique), donc fréquentielle et temporelle.

Un livre de référence sur l'analyse des œuvres musicales et de leurs structures précise que :

[l]'analyse musicale est la résolution d'une structure musicale en éléments constitutifs relativement plus simples, et la recherche des fonctions de ces éléments à l'intérieur de cette structure. (Bent \& Drabkin, 1998 : 9)

Dans le prolongement de celle-ci, nous citons deux définitions dites « organicistes » de la forme musicale, caractéristiques du XIX et du début du XX ${ }^{\mathrm{e}}$ siècle. L'une vient de l'Oxford Companion to Music, dictionnaire musical internationalement réputé :

On entend par « forme » le mode d'organisation de différents éléments d'une œuvre musicale - ses hauteurs, ses rythmes, sa dynamique, son timbre -, dans le but de créer une unité cohérente pour l'auditeur. Depuis des siècles, la définition du mot forme a été le sujet de nombreux débats esthétiques, et dans le contexte musical, la forme ne peut pas être séparée du contenu. (Arnold \& Dunsby 2016 ; notre traduction)

H. Riemann, le théoricien du XIX $X^{\mathrm{e}}$ siècle, souligne également l'unité de l'œuvre : la forme, comme un tout homogène, atteint sa plénitude quand elle est la résultante des oppositions, des contrastes, des conflits (v. Riemann cité par Souris, [1961] 1976 : 248).

En 1967, A. Schoenberg, le compositeur et pédagogue exceptionnel, définit la forme musicale comme suit :

1. Voir, à ce sujet, entre autres, l'ouvrage de référence de Goehr ([1992] 2018) récemment traduit en français. 
Au sens esthétique, le mot «forme » désigne une pièce organisée, c'est-à-dire dont les éléments fonctionnent comme ceux d'un organisme vivant. [...] Les exigences fondamentales préalables à toute création d'une forme intelligible sont la logique et la cohérence. (Schoenberg, [1967] 1987 : 15)

Malgré la référence à l'organicisme et en évoquant, sans le nommer, J. W. von Goethe et ses théories de la morphogenèse végétale (Goethe [1790] 1975), la description des cadres musicaux formels est cantonnée, jusqu'à nos jours, dans la catégorie scientifique dite "mécaniciste ${ }^{2}$. Les principales catégories de formes musicales du passé se fondent sur le mode de la juxtaposition des parties. V. Karbusicky (1990), le sémioticien peircien de la musique, a présenté les cinq archétypes formels qui couvrent 1 'histoire musicale occidentale (et même, parfois, les formes découvertes récemment en zoo-musicologie) :

A. Les formes énumératives [Reihenform] : 1. A A' $\mathrm{A}^{\prime \prime \prime}$ etc. ; 2. Les formes rondeau: ABACAD etc. ; 3. la " production infinie » : ABCD, etc. ;

B. Les formes dites $d^{\prime}$ " équilibre » [Gleichgewichtsform] : 4. les formes palindromiques : $\mathrm{ABA}^{\prime}, \mathrm{ABCB}^{\prime} \mathrm{A}^{\prime}$ etc. ;

C. Les diverses formes dites "évolutives » [Entfaltungsform] : 5. la dramaturgie complexe en quatre actes : forme sonate et forme cyclique superposées.

[cf. Fig. 1 infra]

Dans les années 40-50, le théoricien belge de la forme sonore, A. Souris, a critiqué cette conception mécaniciste de la forme musicale, en s'inspirant de la Gestalttheorie. Il s'appuyait sur les thèses introduites en France par P. Guillaume (1937) et a considéré « la globalité de la forme » comme une donnée première. Cette théorie correspond, dans l'histoire de la musique, à l'émergence de la grande forme chez le dernier Beethoven et chez F. Liszt, forme qui serait déterminée essentiellement par sa finalité, et que l'on appellera " forme téléologique » (cf. la forme archétypique V, Fig. 1 infra).

Un peu plus tard, dans le prolongement des travaux de R. Ruyer (1958) et de ceux de G. Bachelard, A. Souris réclame une vision dynamique de l'approche des formes musicales, empruntée aussi aux découvertes en biologie et en physique, bien au-delà du Gestaltisme. Il parle de la relativisation des facteurs forts et faibles qui définissent la particularité de la forme. Il anticipe, en quelque sorte, les idées de R. Thom sur la théorie des catastrophes, en parlant des processus et de la transformation subite et inattendue de la qualité, déclenchée par le changement survenu dans la quantité :

Il se produit en musique des phénomènes comparables à ceux du monde physique. Un accroissement ou un décroissement de quantité peut, à un certain moment, déclencher une transformation subite de la qualité. (Souris, 1976 : 33)

En 1971, A. J. Greimas annonce, lui aussi, dans sa conférence sur «Les Problèmes généraux de la sémiotique », que, dans les sciences de l'homme,

2. Cf. les entrées des formes dans les grands dictionnaires (Oxford, Grove, MGG, etc.). 
on découvre deux concepts contradictoires et complémentaires, à savoir le " système » et le « processus » :

Chaque système est chargé de sens, et ne peut être considéré comme existant que s'il se manifeste au moyen de processus, d'actions, d'actes réels concrets, d'une production concrète. (Greimas, [1971] 2017 : 40-41 ; nous soulignons)

Les phrases qui suivent expliquent que les sciences de l'homme doivent avoir la même approche que celles utilisées dans la biologie ou dans la physique.

Nous savons que la théorie est toujours en retard sur l'art, sur la pratique. Quand A. Souris et A. J. Greimas ont suggéré une approche " créative » de la théorie des formes, ils ne pouvaient pas savoir que, dès les années 70, la création des nouvelles formes musicales était en marche sous l'inspiration des méthodes $\mathrm{d}^{\prime}$ analyse du son et des phénomènes naturels ${ }^{3}$. D'autres compositeurs ${ }^{4}$ étudiaient les ouvrages scientifiques contemporains. T. Murail et P. Dusapin s'intéressaient à la géométrie fractale de B. Mandelbrot et de J. Gleick ; C. Miereanu à la théorie des catastrophes et aux structures sémio-narratives dans l'interprétation de J. Petitot et d'A. J. Greimas ${ }^{5}$; F.-B. Mâche aux archétypes (phénotypes et génotypes) trouvés dans la nature et dans la zoo-musicologie ; tout récemment P. Manoury et A. Posadas ont découvert et appliqué à leurs formes le L-Système ${ }^{6}$; d'autres compositeurs ${ }^{7}$ approfondissent l'étude du style narratif et de celle des mythes, etc. ${ }^{8}$.

Depuis peu, dans le cadre des publications de l'École sémiotique de Paris, d'importants ouvrages voient le jour sur les approches sémiotiques et philosophiques des images (Dondero \& Fontanille 2012 ; Beyaert-Geslin \& Dondero 2014). Ces recherches riment d'une manière étonnante avec les explorations des modèles scientifiques et visuels pratiquées par les compositeurs contemporains.

$C^{\prime}$ est en ce sens que la sémiotique structurale d'A. J. Greimas rencontre de très près - depuis les années 70-80 - la composition musicale sous le signe du renouvellement, de l'innovation des formes et des contenus sonores.

3. Cf. le naturalisme sonore de Mâche à partir de 1969 et la musique spectrale après 1974.

4. Comme Murail, Miereanu, Dufourt, Lindberg, Manoury, Dusapin, etc.

5. Miereanu a soutenu sa thèse à l'EHESS sous la direction de Greimas en 1979. Son livre publié en 1995, dédié à Greimas, témoigne de l'influence de son maître (l'interface sémiotique-musique : structures profondes, structures superficielles, etc.).

6. Modélisation mathématique de la croissance des plantes, élaborée par Lindenmayer.

7. Comme par exemple Dhomont, Dusapin, Mantovani, Hersant, Pesson, Schoeller, Saariaho, Eötvös, entre autres.

8. D’après Beckett, Kafka, Heiner Müller, Faust, Homère, les romans policiers, etc. 


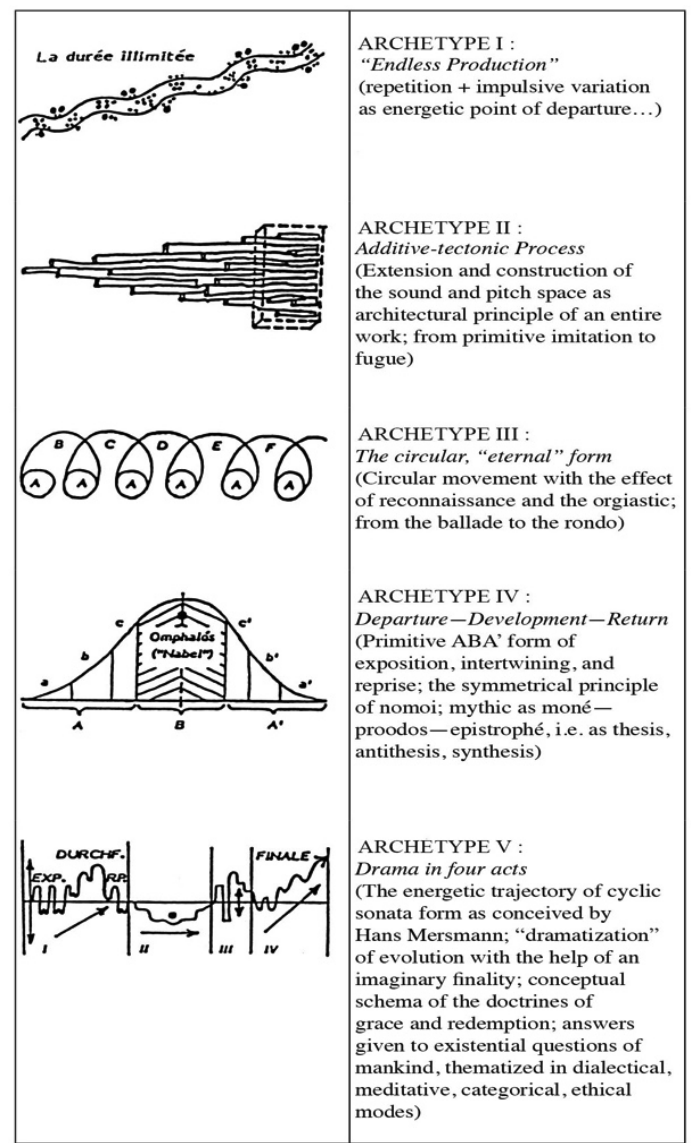

Figure 1 : Les cinq formes archétypiques présentées par Karbusicky (1990 : 195)

\section{3. "STRUCTURE " VERSUS "SIGNIFICATION " : LA GUERRE ENTRE FORMALISME (OU “ NIHILISME ESTHÉTIQUE ") ET ESTHÉTIQUE DU CONTENU EN MUSIQUE}

Le conflit entre les formalistes et les représentants de l'esthétique du contenu ${ }^{9}$ en musique existe depuis l'Antiquité. À chaque période historique, la confrontation se présente sous des apparences nouvelles comme, par exemple, au tout début quand les Pythagoriciens se confrontent à Aristote et Platon. Au XVIII ${ }^{\mathrm{e}}$ siècle, ce dualisme privé de médiation correspond à la séparation de deux tendances dans l'histoire de la philosophie : le positivisme et l'idéalisme transcendantal ou subjectif (son reflet en musique serait le conflit entre Rameau et Rousseau). Au

9. Termes des théoriciens et des compositeurs des $\mathrm{XIX}^{\mathrm{e}}-\mathrm{XX}^{\mathrm{e}}$ siècles. 
milieu du XIX $X^{\mathrm{e}}$ siècle, la scission entre les deux modes $\mathrm{d}^{\prime}$ approches atteint son paroxysme : on assiste à la naissance de la musique « absolue » ou " autonome » (cf. Hanslick) et à celle de la « musique à programme » (cf. Liszt et Berlioz) ${ }^{10}$.

De nos jours, avec l'avènement de la sémiotique musicale, on constate toujours l'affrontement ou l'antagonisme entre ces deux modes d'approche : une sémiose ou une sémiotique musicale dite "immanente " ${ }^{11}$ et une sémiotique musicale construite sur le concept de la « signification musicale » depuis la création du projet international ICMS (International Conference on Musical Signification) en 1984 à Paris, sous l'influence d'E. Tarasti, de D. Charles et des élèves d'A. J. Greimas ${ }^{12}$.

La sémiotique musicale, que je qualifierais de "formaliste », est née sous l'influence de J. Molino, avec sa tripartition appliquée à la musique ${ }^{13}$ par J.-J. Nattiez (1975). O. Kühl formule d'une manière intéressante le problème inhérent à cette sémiologie de la musique « sans signes » :

I regard Nattiez's attempt to build a semiology without signs as primarily a way of legitimizing old-fashioned musical hermeneutics, and accordingly unable to contribute much towards a cognitive musicology. Nattiez's theories remain ungrounded in cognitive and neurobiological empirical studies. (Kühl, $2007: 228{ }^{14}$ )

Dans les années 80-90, la sémiotique musicale gagne du terrain aux ÉtatsUnis également. Un des points de départ de l'approche sémiotique de R. Hatten, par exemple, était la théorie du marquage d'après $M$. Shapiro. Elle introduit l'approche binaire dans la production du sens lors de l'analyse d'une forme musicale. Les méthodes d'A. J. Greimas utilisées en musique par les sémioticiens européens - isotopie, sème, binarité, carré sémiotique [avec contrariété, négation, etc.], trajectoire ou programme suivant un axe euphorique ou dysphorique, etc. - parviennent aux mêmes résultats que les musicologues anglosaxons, partant de la théorie du marquage, tout en arrivant aux "stratégies narratives » ou « stratégies de genres expressifs».

Toujours dans les années 90, le sémioticien et esthéticien anglais de la musique, $R$. Monelle, a présenté longuement la méthode greimassienne (Monelle, 1992 : 220-274), mais il a également introduit la critique et le commentaire d'une sémiotique musicale positiviste dangereuse. "À ses débuts, dans sa version Nattiez-Molino, la sémiotique musicale avait elle-même pris la forme d'une tentative de mise au ban de la sémantique » (id., $2016: 29$ ). R. Monelle offre une

10. Voir Fubini (2000) et Ujfalussy (1980).

11. Termes de Meeùs et Nattiez dans le cadre d'une sémiotique formaliste !

12. Tels que Miereanu, Stoianova, Castellana; plus tard, Grabócz, Vecchione, Stefani, Esclapez, Jacono, Hauer, etc.

13. Niveaux d'approches séparés en niveaux neutre, poḯtique et esthésique.

14. Et l'auteur rajoute en note de bas de page : « Nattiez explicitly states that 'semiology is not the science of communication' » (loc. cit.). 
caricature, sous le nom du Dr. Strabismus, d'un musicologue qui cherche à échafauder une théorie générale, une totalisation encyclopédique, et qui n'arrive qu'à une collection d'ébauches :

Une théorie générale n'était plus possible. Seul un accent sur le sens de la musique, plutôt que sur ses formes ou sa syntaxe, avait uni ses pensées aléatoires. Il se trouvait dans ce que l'on a appelé la condition postmoderne. (Monelle, 2016 : 17)

Actuellement, dans les territoires francophones, ce sont toujours deux sémiotiques musicales qui s'affrontent quotidiennement ${ }^{15}$ : la sémiotique formaliste autour de J.-J. Nattiez, N. Meeùs ${ }^{16}$, S. Badir, et la sémiotique musicale greimassienne qui s'intéresse à l'articulation du sens, des signifiés, aux programmes narratifs en tant qu'une couche supplémentaire d'analyse qui complète et parfait l'examen des formes et des structures en musique. Ce deuxième groupement de sémioticiens de la musique se réunit actuellement autour d'E. Tarasti et de son projet d'ICMS (après la disparition de D. Charles qui le soutenait et y collaborait jusqu'en 2008). Ce programme de recherche international consiste en l'organisation de congrès internationaux bisannuels à travers toute l'Europe ${ }^{17}$ et s'appelle Projet international de la Signification Musicale ; les congrès sont accompagnés de publications des actes de colloque ${ }^{18}$.

\section{LA QUESTION DU SENS ET DE LA SIGNIFICATION EN MUSIQUE : APPORT DE LA SÉMANTIQUE STRUCTURALE, DE LA SÉMIOTIQUE, DE LA GRAMMAIRE NARRATIVE DE GREIMAS À LA COMPRÉHENSION ET LA CONNAISSANCE DES EUVRES MUSICALES}

L'auteur de ces lignes s'est engagée auprès des méthodes greimassiennes depuis 1988 , tout en renouvelant constamment la panoplie des méthodes sémiotiques du maître appliquées à la musique. Cette adhésion assumée s'explique aussi par le fait que les grands musicologues hongrois et ceux des pays de l'Est

\footnotetext{
15. Cf. par exemple la présentation des théories de sémiotique formaliste et de celles greimassiennes lors du $9^{\mathrm{e}}$ Congrès européen de l'Analyse musicale - 9th EUROMAC -, congrès tenu à Strasbourg entre les 28-06 et 01-07-2017.

16. Son article de 2009 offre une critique approfondie de certaines notions greimassiennes utilisées en musique (telles que l'« isotopie » et le «carré sémiotique »). Son texte de 2001 affirme que « le signifiant [...] ne peut lui-même être un objet matériel. [...] La première opinion, selon laquelle un signifiant matériel renvoie à un signifié conceptuel, doit être rejetée pour des raisons de simple logique [...]. Mais la seconde conception, qui situe tant le signifiant que le signifié au niveau mental - donc individuel - ne permet pas d'éclairer la sémiose comme phénomène social » (Meeùs, $2001: 554$ ).
}

17. En changeant de ville et d'université tous les deux ans, selon les candidats se proposant parmi les grands centres européens de la musique (p. ex. Helsinki, Imatra, Édimbourg, Paris, Bologne, Aix-en-Provence, Rome, Londres, Canterbury, Cracovie, Louvain, Bruxelles, etc.).

18. En mai 2018 fut organisé, à la suite de ses débuts parisiens de 1984, le 14 $4^{\mathrm{e}}$ Congrès d'ICMS, coordonné par Tarasti \& Andreica au Conservatoire G. Dima de Cluj-Napoca (Roumanie). 
des années 60-80 ${ }^{19}$, tout comme A. J. Greimas, ont considéré l'art comme un phénomène social. Ils ont considéré le discours artistique, le récit ou le discours musical comme porteurs de valeurs au sein d'une société. Les « discours » des grands compositeurs (comme ceux des grands écrivains) ont été souvent lus, écoutés, analysés par ces maîtres comme des messages adressés aux membres de la communauté culturelle de leur pays, messages qui incitent à réfléchir sur des questions de valeurs d'une société.

En parlant des récits (de toutes catégories) et de leurs formes d'expression de surface qui dissimulent chacune un sens profond, A. J. Greimas précise :

C'est le déchiffrement de ces récits (des œuvres littéraires de création individuelle ou collective, du folklore) qui nous permet de découvrir les systèmes de valeurs qui y sont dissimulés et les résumés du comportement selon ces systèmes de valeurs, les idéologies. (Greimas, [1971] 2017 : 44)

Dans ce même article, en évoquant G. Bernanos, A. J. Greimas parle également des modèles ou des opérations logiques qui permettent de détecter ou de « résoudre les changements du contenu dans l'œuvre » :

[...] Il existe une certaine dialectique, une dialectique logique qui permet de comprendre les transformations de l'idéologie de l'ouvrage, les transformations de l'ouvrage [...]. (Greimas, [1971] 2017 : 51)

Dans les limites de cette contribution, il serait impossible de tenir compte et de faire l'inventaire de tous les apports de la sémiotique greimassienne à la musicologie. Je vais restreindre ma présentation (i) aux unités correspondant aux signifiés ; (ii) à quelques modèles choisis pour expliquer l'apport d'A. J. Greimas à la compréhension des stratégies narratives (ou des stratégies de l'organisation des signifiés) dans les œuvres musicales de plusieurs périodes.

\subsection{Terminologie concernant les signifiés en musique}

Depuis la période baroque (où les traités répertoriaient les figures de rhétorique musicale, puis les «madrigalismes »), on tourne consciemment vers les unités musicales signifiantes dans les années 30-40, à l'époque de B. Asafiev (contemporain de V. Propp et de J. Tynianov), qui a introduit le terme d'intonation (d'après J.-J. Rousseau) pour parler des formules musicales caractéristiques (du point de vue de leurs paramètres musicaux) ayant une expression spécifique, qui transmettent un sens humain ou social, et qui représentent des caractères déterminés au sein d'une pièce musicale (v. Ujfalussy, cité par Grabócz, 2009 : 45-46). La catégorie d'intonation entre également en contact avec « le typique » dans le sens où les figures d'un style sont reconnues de tous, en tant qu'éléments d'un réservoir, d'un memoranda collectif. (v. aussi Tarasti, 2006 : 81-82 ; Monelle,

19. Musicologues qui avaient encore une connaissance quasi encyclopédique de l'histoire de la musique, tels que Szabolcsi, Ujfalussy, Asafiev, Karbusicky; puis, ceux de la génération suivante : Maróthy, Jiránek, Kroó, Somfai, Tallián, etc. 
1992 : 274-303). À partir des années 80, E. Tarasti et d'autres musicologues ${ }^{20}$ ont

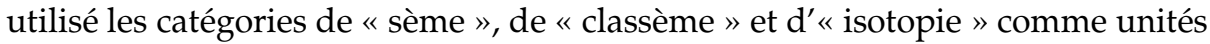
correspondant aux signifiés en musique (cf. les définitions de ces termes dans Grabócz 1996). En 1980, L. Ratner publie aux États-Unis le premier ouvrage dans lequel la notion de « topique musical » est introduite en tant qu'élément typique possédant une expression reconnue de tous à l'époque classique ${ }^{21}$. Cette notion correspond à celle $\mathrm{d}^{\prime}$ " intonation » de $\mathrm{B}$. Asafiev mais aussi à celle de "classème " (grandeurs ou longueurs couvrant un thème musical, i.e. environ huit ou seize mesures). À partir des années 2000-2010, c'est le terme de topique qui va dominer l'analyse de la signification en musique, au détriment des autres notions (bien que celui d'isotopie musicale garde toujours sa valeur en tant qu'unité de plus grande extension ou combinant d'autres unités). De nos jours, c'est l'Oxford Handbook of Topic Theory, publié en 2014 sous la direction de D. Mirka, qui rassemble le répertoire le plus complet des topiques musicaux recensés : environ 150 items depuis le Baroque jusqu' au $\mathrm{XX}^{\mathrm{e}}$ siècle ${ }^{22}$.

\subsection{Articulation du sens et des signifiés à l'intérieur des formes musicales}

La deuxième question de nature sémiotique, impliquée par l'analyse des formes en musique, concerne la stratégie de l'organisation des signifiés. Les musicologues ont eu recours à plusieurs modèles d'A. J. Greimas : le plus souvent au carré sémiotique pour présenter l'articulation complexe des signifiés musicaux à l'intérieur d'une pièce ${ }^{23}$. Certaines applications du " carré de Greimas » sont des schèmes "statiques ", d'autres introduisent la temporalité, l'évolution et la transformation dans la représentation des formes à l'aide de la structure élémentaire de la signification. Voici un exemple «dynamique » du carré (Fig. 2 infra).

Un autre modèle, souvent utilisé chez les musicologues, est le parcours génératif d'A. J. Greimas avec ses deux niveaux principaux - niveau de surface et niveau profond - et sa syntaxe discursive (actorialisation, temporalisation, spatialisation). L'analyse synthétique et exemplaire d'E. Tarasti du premier mouvement de la sonate Waldstein op. 53 de L. van Beethoven explore tous les aspects de cette syntaxe (Tarasti, $1996:$ :76-103). La formule du programme narratif d'A. J. Greimas a été également souvent exploitée, avec son sujet d'état, sujet de faire, l'énoncé de faire, l'énoncé d'état, le sujet relié, ou non, à un objet de valeur, etc. (cf. Fig. 3 infra).

20. Stoianova, Miereanu, Grabócz, Cassar, Desquilbé, Ellis, entre autres.

21. Terme utilisé dans sens du 'lieu commun'.

22. Les topiques du XVIII ${ }^{\mathrm{e}}$ siècle sont, par exemple, le pastoral ; le solennel ; le tragique, le grotesque ; la chasse ; les styles de danse portant une signification : gagliarda, polonaise, siciliano, etc.

23. Cf. Tarasti, Grabócz, Poirot, Mirka, Sivuoja-Gunaratnam, Everett, Stepien, Jankauskienè, Pawlowska, Grimalt, etc. 


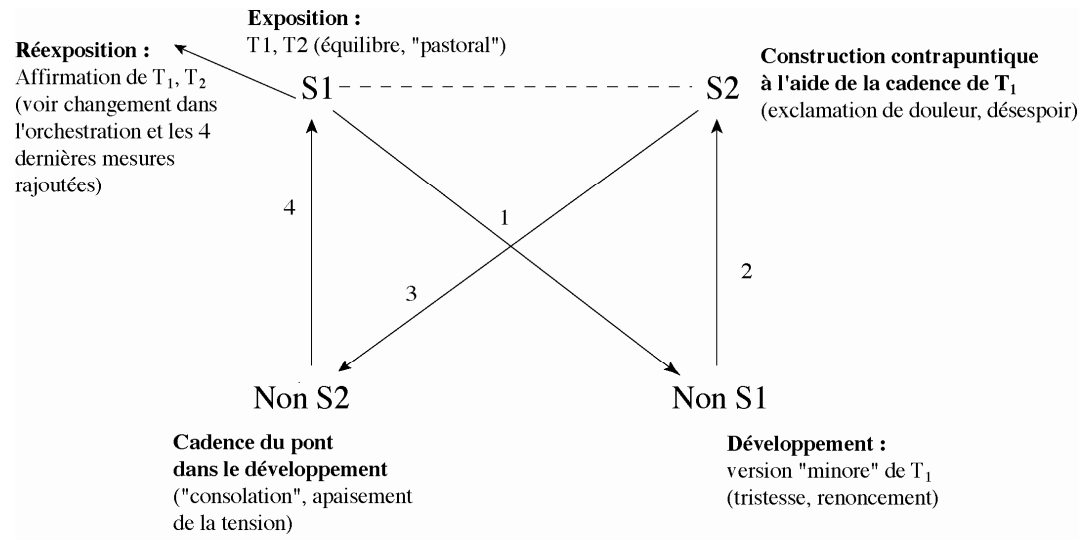

Figure 2 : Schéma global de l'articulation des sèmes (ou affects, topiques) dans

l'Andante de la Symphonie K. 504 dite « de Prague » de Mozart [les chiffres sur les lignes correspondent à l'évolution, aux étapes dans le déroulement de la forme sonate]

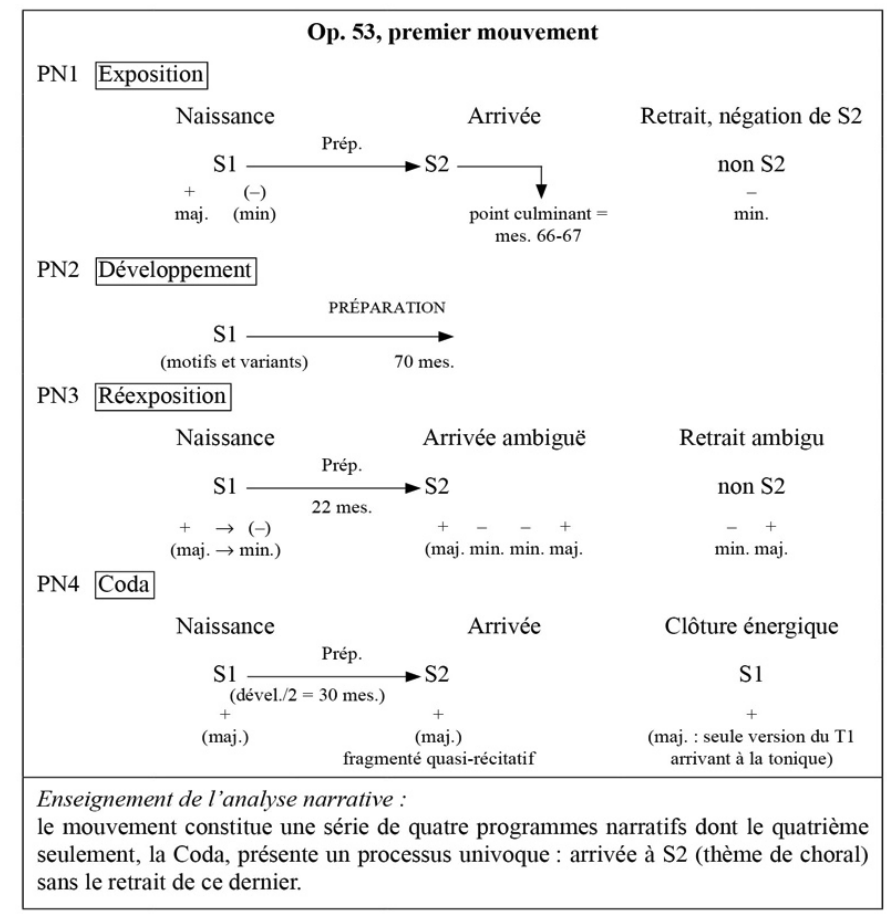

Figure 3 : Schéma de l'évolution des signifiés dans le premier mouvement de la sonate Waldstein op. 53 de Beethoven en quatre programmes narratifs [Grabócz, 2009 : 188] 
Ce mouvement de L. van Beethoven utilise quatre programmes narratifs et ce n'est seulement le dernier (PN4) qui offre la succession euphorique de S1-S2, suivie d'une arrivée aboutie et non annulée (comme dans les précédents PN).

Un autre usage précoce et vraiment révolutionnaire pour l'analyse musicale fut l'introduction du concept de série des programmes narratifs dans l'analyse de la Polonaise-Fantaisie op. 61 de F. Chopin par E. Tarasti ([1987] 1996).

\begin{tabular}{|c|c|c|}
\hline PN 1 & S'enfoncer / se relever (mes. 1-8) & -+ \\
\hline PN 2 & Naissance du thème principal ([Polonaise] : mes. 9-21) & - \\
\hline PN 3 & Polonaise /version 1 (mes. 22-60) & + \\
\hline PN 4 & Modulations ou débrayage topologique (mes. 67-91) & - \\
\hline PN 5 & Polonaise/version 2 : états thymiques extrêmes (mes. 92-115) & + \\
\hline PN 6 & Mazurka/version 1 (mes. 116-147) & + \\
\hline PN 7 & Nocturne/ version 1 (mes. 153-180) & - \\
\hline PN 8 & Mazurka/version 2 (mes. 181-198) & + \\
\hline PN 9 & \begin{tabular}{ll}
\multicolumn{2}{l}{ Eloignement et retour (mes. 199-240) } \\
- & Nocturne / 2 (mes. 201) si majeur \\
- & Introduction variée (mes. 205-214) ré majeur, do majeur \\
- & Mazurka / 3 (mes. 215) fa mineur \\
- & Transition: (mes. 225) « a tempo primo » fa mineur \\
\end{tabular} & -- \\
\hline PN 10 & $\begin{array}{ll}\text { Accomplissement (mes. 241-288) } \\
-\quad & \text { Polonaise / } 3: \text { (mes. 242) la bémol majeur } \\
- & \text { Transition (variation de Mazurka) (mes. 248) si majeur } \\
- & \text { Nocturne / } 3 \text { (mes. 253) } \\
- & \text { Polonaise + Mazurka + Nocturne superposés (mes. } 267 \text { et } \\
\text { suivantes) : accelerando, sempreff la bémol majeur }\end{array}$ & ++ \\
\hline
\end{tabular}

Figure 4 : Analyse de la Polonaise-Fantaisie de Chopin (op. 61) par Tarasti en 10 programmes narratifs (Tarasti, [1987] 1996 : 195-216) [ce schéma a été rédigé par Grabócz et complété des marques d'euphorie et de dysphorie]

Un autre modèle de la théorie greimassienne, qui a été souvent utilisé par les musicologues, est la théorie des modalités (Tarasti dans Chopin ; Stoianova, Pankhurst, Heimonen dans Liszt, etc. ; cf. Tarasti, 1996 : 217-254).

\section{CONCLUSION}

Comme j'ai essayé de le souligner supra, la musicologie traditionnelle est quasiment handicapée en ce qui concerne la compréhension et l'analyse complexe des morceaux instrumentaux dramatiques (cathartiques) de l'époque classique, d'un grand nombre d'œuvres symphoniques romantiques et des dramaturgies musicales élaborées ou sophistiquées des $\mathrm{XX}^{\mathrm{e}}-\mathrm{XXI}^{\mathrm{e}}$ siècles. Les compositeurs 
eux-mêmes réfléchissent le plus souvent sur le contenu d'abord, et la forme, $l^{\prime}$ expression de surface s'adapte aux exigences du propos, du message ${ }^{24}$.

Outre le fait que la recherche des sèmes, des classèmes et d'isotopies (ou des topiques et intonations) assure un lien avec les unités culturelles de la société, et de l'époque dans laquelle l'œuvre est produite, la grande forme d'une pièce musicale cache souvent une évolution de type binaire des pathèmes, des affects.

Les méthodes d'analyse sémiotique textuelle d'A. J. Greimas ont éclairé les formes cathartiques mozartiennes ou beethovéniennes, car, en mettant le doigt sur les relations de contrariété ou de contradiction, sur la relation de complémentarité au niveau des topiques, on constate la transformation, voire la création des " nouveaux objets de valeur » à l'intérieur du discours musical, et ces nouveaux objets de valeur, ces nouvelles idées musicales, poussent la forme à sortir de ses cadres habituels. Ce qui veut dire, du point de vue de l'analyse des styles musicaux, que ces pièces de l'époque classique contiennent en elles-mêmes l'essence de la structure romantique : la "forme téléologique", qui crée de nouvelles valeurs tout au long du discours musical ${ }^{25}$.

Dans la musique contemporaine, nous assistons à un renouveau du concept de « la musique à programme » du XIX ${ }^{\mathrm{e}}$ siècle, dans le sens où les compositeurs choisissent consciemment des modèles narratifs, visuels, scientifiques, astrophysiques, psychologiques, ou autres, pour «communiquer» avec le public de nos jours. Si le musicologue n'est pas enclin à analyser structuralement ces " objets sémiotiques nouveaux », la compréhension des nouvelles musiques lui échappera de façon assurée.

Les méthodes d'A. J. Greimas ont également fait leur preuve dans l'analyse interdisciplinaire, plus précisément dans l'interface entre musique et littérature, dans la mesure où la structuration de la signification n'est pas strictement déterminée par le type d'expression artistique. Nous pouvons évoquer la comparaison des mythes avec les poèmes symphoniques du XIX ${ }^{\mathrm{e}}$ siècle par E. Tarasti (2003) ; l'analyse de la forme canonique lisztienne en quatre étapes (la succession de quatre isotopies obligatoires) et l'analyse structurelle en quatre sections du Faust de J. W. von Goethe produite par les chercheurs en littérature et en philosophie (Grabócz, 2009 : 249-260, 221-248).

On ne peut que souhaiter qu'un jour la jeune génération évalue l'apport complet de la sémiotique greimassienne à la musicologie, en se penchant sur la trentaine d'ouvrages-clé qui a marqué l'histoire de la sémiotique musicale européenne de 1980 à nos jours.

24. Cf. l'une des premières tentatives de recensement de l'influence des théories scientifiques récentes sur l'innovation des formes musicales contemporaines dans Grabócz (2016).

25. Cf. par exemple, les analyses de certaines œuvres dramatiques, devenues « téléologiques », chez Mozart et Beethoven dans Grabócz (2009 : 111-130, 131-165) ; l'analyse de la Fantaisie en ré mineur K.397 de Mozart présentée en programmes narratifs expressifs par Tarasti $(2016: 65-81)$. 


\section{Références}

Agawu K. (1991), Playing with Signs: A Semiotic Interpretation of Classic Music, Princeton, Princeton University Press.

ARnold D. \& DunsBy J. (2016), “Form”, in A. Latham (ed.), The Oxford Companion to Music. Oxford Music Online, Oxford University Press. [disponible en ligne]

BENT I. \& DRABKIn W. ([1980] 1998), L'analyse musicale : histoire et méthodes, Paris, Éditions Main d'œuvre.

BeyAert-Geslin A. \& Dondero M. G. (éds) (2014), Arts et sciences : approches sémiotiques et philosophiques des images, Liège, Presses Universitaires de Liège.

DANUSER H. (ed.) (1998²), Gattungen der Musik und ihre Klassiker, Regensburg, Laaber-Verlag.

Dondero M. G. \& FontANILLE J. (2012), Des images à problèmes : le sens du visuel à l'épreuve de l'image scientifique, Limoges, Pulim.

FuBINI E. (2000), Les philosophes et la musique, Paris, Slatkine Reprints.

GoEHR L. ([1992] 2018), Le musée imaginaire des œuvres musicales, Paris, Cité de la MusiquePhilharmonie.

GOETHE J. W. von ([1790] 1975), La métamorphose des plantes, Paris, Triades.

Grabócz M. (1996), "The role of semiotic terminology in musical analysis ", in E. Tarasti (ed.), Musical Semiotics in Growth, Bloomington, Indiana University Press, 195-218.

Grabócz M. (éd.) (2007), Sens et signification en musique, Paris, Hermann.

GRABócz M. (2009), Musique, narrativité, signification, Paris, L'Harmattan.

Grabócz M. (2016), "Formes musicales sous l'influence des théories scientifiques dans les œuvres contemporaines ", dans C. Maeder \& M. Reybrouck (éds), Sémiotique et vécu musicale : du sens à l'expérience, de l'expérience au sens, Actes du congrès ICMS 12, Leuven, Leuven University Press, 113-134.

GREIMAS A. J. ([1971] 2017), "Les problèmes généraux de la sémiotique (1971) ", dans Du sens en exil : chroniques lithuaniennes, textes présentés par I. Darrault-Harris \& D. Bertrand, traduit du lithuanien par L. Perkauskyté, Limoges, Lambert-Lucas, 37-58.

GreimAs A. J. \& CouRTÉs J. (1979-1983), Sémiotique : dictionnaire raisonné de la théorie du langage, 2 vol., Paris, Hachette.

GUILLAUME P. (1937), La psychologie de la forme, Paris, Flammarion.

HAtTEn R. (1994), Musical Meaning in Beethoven: Markedness, Correlation, Interpretation, Bloomington, Indiana University Press.

HATTEN R. (2004), Interpreting Musical Gestures, Topics, and Tropes, Bloomington, Indiana University Press.

KARBUSICKY V. (1990), Kosmos-Mensch-Musik: Strukturalistische Anthropologie des Musikalischen, Hamburg, Verlag Dr. R. Krämer.

KüHL O. (2007), Musical Semantics, Berlin, Peter Lang.

MeEùs N. (2001), "Le statut sémiologique de l'analyse musicale ", dans J.-M. Chouvel et alii (éds), Analyse et création musicales, Paris, L'Harmattan, 549-562. [disponible en ligne]

Meeùs N. (2009), "L'autonomie de la sémiotique ", dans C. Hauer \& B. Vecchione (éds), Le sens langagier du musical : sémiosis et hermenéia, Paris, L'Harmattan, 187-196. [disponible en ligne]

Miereanu C. (1995), Fuite et conquête du champ musical, Paris, Méridiens-Klincksieck.

MiRKA D. (ed.) (2014), The Oxford Handbook of Topic Theory, Oxford, Oxford University Press. 
Monelle R. (1992), Linguistics and Semiotics in Music, Chur, Harwood Academic Publishers.

MonelLE R. ([2000] 2016), Un chant muet : musique, signification, déconstruction, Paris, La Rue Musicale / Philharmonie de Paris.

NATtIEZ J.-J. (1975), Fondements d'une sémiologie de la musique, Paris, Union générale d'éditions, 10/18.

RATNer L. (1980), Classic Music: Expression, Form and Style, New York, Schirmer.

RUYER R. (1958), La genèse des formes vivantes, Paris, Flammarion.

Schoenberg A. ([1967] 1987), Fondements de la composition musicale, tr. D. Collins, Paris, J.-C. Lattès.

SHeInBERg E. (ed.) (2012), Music Semiotics: A Network of Significations: In Honour and Memory of Raymond Monelle, Ashgate, Aldershot.

SouRIS A. ([1961] 1976), "Sur quelques termes fondamentaux du vocabulaire musical - Forme ", dans A. Souris (éd.), Conditions de la musique et autres écrits, Bruxelles/Paris, Université de Bruxelles/CNRS Éditions, 248-253.

TARAStI E. (ed.) (1996), Musical Semiotics in Growth, Bloomington, Indiana University Press.

TARAStI E. ([1978] 2003), Mythe et musique : Wagner, Sibelius, Stravinsky, trad. D. Pousset, Paris, Michel de Maule.

TARASTI E. (2006), La musique et les signes : précis de sémiotique musicale, Paris, L'Harmattan.

TARASTI E. (2016), Sémiotique de la musique classique : comment Mozart, Brahms et Wagner nous parlent, trad. L. Csinidis \& M. Rousselot, Aix-en-Provence, Publications de I'Université de Provence.

UjFalussy J. (1968), Az esztétika alapjai és a zene [Les Fondements de l'esthétique et la musique], Budapest, Tankönyvkiadô.

UjFaLussy J. (1980), "Zene és valóság " [Musique et réalité], dans Zenéröl, Esztétikláról [Sur la musique, sur l'esthétique], Budapest, Zenemükiadó, 150-179. 